\title{
On the Classification of Subalgebras of the Galilei Algebras
}

\author{
Leonid BARANNYK \\ Department of Mathematics, Pedagogical Institute, \\ 2 Ostrogradsky Street, 314003 Poltava, Ukraina
}

\begin{abstract}
We investigate the structure of certain types of subalgebras of Galilei algebras and the relationship between the conjugacies of these subalgebras under different groups of automorphisms.
\end{abstract}

\section{Introduction}

In the following, we use the terms "full, special, extended, and classical Galilei algebra of an $n$-dimensional space" for the optical algebra of the Minkowski space $\mathbf{R}_{1, n+1}$, the extended Schrödinger algebra, the extended Galilei similitude algebra, and the extended Galilei algebra of an $n$-dimensional space, respectively. We use the following notation for these algebras:

$$
\begin{array}{ll}
A G_{4}(n)=A O p t(1, n+1), & A G_{3}(n)=A \widetilde{S c h}(n), \\
A G_{2}(n)=A \tilde{G}(n) \boxplus<D>, & A G_{1}(n)=A \tilde{G}(n) .
\end{array}
$$

The reduced algebra corresponding to $A G_{j}(n)$ is the factor algebra $A \bar{G}_{j}(n)=$ $=A G_{j}(n) /<M>$.

The classification of subalgebras of the Galilei algebras for $n=2,3$, was done in [1-7]. Subalgebras of the Galilei algebras for an arbitrary $n$-dimensional space were investigated in $[4,7-9]$. In $[7,10]$ the reduction to ordinary differential equations of the linear heat equation was carried out, using $I$-maximal subalgebras of the algebra $A G_{3}(n)$. The symmetry reduction by all $I$-maximal subalgebras of the corresponding Galilei algebras for different nonlinear generalizations of the Schrödinger equation was done in $[7,11]$. Reducing hyperbolic equations to parabolic ones [12], one can exploit subalgebras of the Galilei algebras in order to construct wide classes of exact solutions of hyperbolic equations. This was done in [12] using subalgebras of $A G_{3}(2)$.

In this article, we give conditions for splittability of all extensions of a subalgebra of the algebra $A O(n) \oplus A G L(2, \mathbf{R})$ in the reduced full Galilei algebra, from which it is easy to obtain the corresponding conditions for other Galilei algebras. We give modified formulations of theorems about the structure of subalgebras of the Galilei algebras [7], which, we believe, are easier to understand and apply. We also investigate the connection between the conjugacies of subalgebras under groups of inner automorphisms of different Galilei algebras. 


\section{Definitions of Galilei Groups and Algebras}

We denote by $A G$ the Lie algebra of the Lie group $G$.

The reduced full Galilei group of $n$-dimensional space is the multiplicative group $\bar{G}_{4}(n)$ of matrices

$$
\left(\begin{array}{ccc}
\Gamma & \vec{v} & \vec{a} \\
0 & \alpha & \beta \\
0 & \gamma & \delta
\end{array}\right),
$$

where $\Gamma \in O(n), \vec{a}, \vec{v} \in \mathbf{R}^{n}, \alpha, \beta, \gamma, \delta \in \mathbf{R}$ and $\alpha \delta-\beta \gamma \neq 0$. The reduced special Galilei group $\bar{G}_{3}(n)$ is a subgroup of the group $\bar{G}_{4}(n)$ which consists of those matrices (1), for which $\alpha \delta-\beta \gamma=1$. The reduced extended Galilei group $\bar{G}_{2}(n)$ is the group of matrices (1) for which $\gamma=0, \delta=\alpha^{-1}$. If $\alpha=\delta=1, \gamma=0$, we obtain matrices that are elements of the reduced classical Galilei group $\bar{G}_{1}(n)$.

The Lie algebra $A \bar{G}_{4}(n)$ of the group $\bar{G}_{4}(n)$ consists of real matrices

$$
\left(\begin{array}{ccc}
X & \vec{v} & \vec{a} \\
0 & \lambda & \mu \\
0 & \nu & \rho
\end{array}\right),
$$

where $X \in A O(n), \lambda, \mu, \nu, \rho \in \mathbf{R}$ and $\vec{a}, \vec{v} \in \mathbf{R}^{n}$. Let $I_{a b}$ be a matrix of order $n+2$ having unity at the intersection of the $a$-th line and the $b$-th column and zeros elsewhere $(a, b=1, \ldots, n+2)$. The matrices

$$
\begin{array}{lll}
J_{a b}=I_{a b}-I_{b a}, & G_{a}=I_{a, n+1}, \quad P_{a}=I_{a, n+2}, & T=I_{n+1, n+2}, \\
S=-I_{n+2, n+1}, & D=-I_{n+1, n+1}+I_{n+2, n+2}, & Z=I_{n+1, n+1}+I_{n+2, n+2}
\end{array}
$$

$(a<b ; a, b=1, \ldots, n)$ form a basis of the algebra $A \bar{G}_{4}(n)$. They satisfy the following commutation relations:

$$
\begin{aligned}
& {\left[J_{a b}, J_{c d}\right]=\delta_{a d} J_{b c}+\delta_{b c} J_{a d}-\delta_{a c} J_{b d}-\delta_{b d} J_{a c} ;} \\
& {\left[P_{a}, J_{b c}\right]=\delta_{a b} P_{c}-\delta_{a c} P_{b} ; \quad\left[G_{a}, J_{b c}\right]=\delta_{a b} G_{c}-\delta_{a c} G_{b} ;} \\
& {\left[P_{a}, P_{b}\right]=\left[G_{a}, G_{b}\right]=0 ; \quad\left[G_{a}, P_{b}\right]=0 ;} \\
& {\left[D, J_{a b}\right]=\left[S, J_{a b}\right]=\left[T, J_{a b}\right]=\left[Z, J_{a b}\right]=0 ;} \\
& {\left[D, G_{a}\right]=G_{a} ; \quad\left[D, P_{a}\right]=-P_{a} ; \quad\left[S, G_{a}\right]=0 ; \quad\left[S, P_{a}\right]=G_{a} ;} \\
& {\left[T, G_{a}\right]=-P_{a} ; \quad\left[T, P_{a}\right]=0 ; \quad\left[Z, G_{a}\right]=-G_{a} ; \quad\left[Z, P_{a}\right]=-P_{a} ;} \\
& {[D, S]=2 S ; \quad[D, T]=-2 T ; \quad[T, S]=D ;} \\
& {[D, Z]=[S, Z]=[T, Z]=0 \quad(a, b, c, d=1, \ldots, n) .}
\end{aligned}
$$

It is easy to verify that $\langle D, S, T\rangle=A S L(2, \mathbf{R})$ and $\langle D, S, T\rangle \oplus\langle Z\rangle=$ $=A G L(2, \mathbf{R})$. Let

$$
Q[a, b]=<P_{a}, \ldots, P_{b}, G_{a}, \ldots, G_{b}>\quad(a \leq b) .
$$

Then

$$
A \bar{G}_{4}(n)=Q[1, n] \oplus(A O(n) \oplus A G L(2, R)),
$$




$$
\begin{aligned}
& A \bar{G}_{3}(n)=Q[1, n] \oplus(A O(n) \oplus A S L(2, R)), \\
& A \bar{G}_{2}(n)=Q[1, n] \oplus(A O(n) \oplus<D, T>), \\
& A \bar{G}_{1}(n)=Q[1, n] \oplus(A O(n) \oplus<T>) .
\end{aligned}
$$

We denote by $A \bar{G}_{0}(n)$ the algebra $Q[1, n] \boxplus A O(n)$.

The full algebra $A G_{4}(n)$ is obtained from the algebra $A \bar{G}_{4}(n)$ by adding the element $M$. We have, moreover, $\left[G_{a}, P_{b}\right]=\delta_{a b} M,[Z, M]=-2 M,[M, X]=0$ for every basis element $X \neq Z$; the other commutation relations in (2) are unchanged. The factor algebra $A G_{4}(n) /<M>$ is identified with $A \bar{G}_{4}(n)$. We shall denote the generators of the algebras $A G_{4}(n)$ and $A \bar{G}_{4}(n)$ by the same symbols.

Let $\Phi(n)=<M, P_{1}, \ldots, P_{n}, G_{1}, \ldots, G_{n}>$. Then

$$
A G_{4}(n)=\Phi(n) \oplus(A O(n) \oplus A G L(2, \mathbf{R})) .
$$

The special, extended, and classical Galilei algebras are obtained analogously.

We shall use the following notations:

$$
\begin{aligned}
& A O[k, l]=<J_{a b}: a, b=k, k+1, \ldots, l>; \\
& V[k, l]=<G_{k}, G_{k+1}, \ldots, G_{l}>; \quad W[k, l]=<P_{k}, P_{k+1}, \ldots, P_{l}>.
\end{aligned}
$$

The group of inner automorphisms of the Lie algebra $L$ is denoted by $A d L$.

Each inner automorphism of the algebra $A O(n)$ can be extended uniquely to an inner automorphism of the algebra $A \bar{G}_{j}(n)(0 \leq j \leq 4)$. In this sense, we say that $A d A O(n)$ is a subgroup of $A d A \bar{G}_{j}(n)$.

The subalgebra $K$ of the algebra $A \bar{G}_{4}(n)$ is said to be split if $K=U \boxplus F$, where $U \subset Q[1, n]$ and $F \subset A O(n) \oplus A G L(2, \mathbf{R})$. We say that $K$ is splittable if there exists an inner automorphism $\psi$ in $A d A \bar{G}_{4}(n)$ such that $\psi(K)$ is split. Splittability of subalgebras of the other Galilei algebras is defined in the same manner. By conjugacy of subalgebras of a Lie algebra $L$, we mean conjugacy under the group $A d L$.

\section{Criterion for the Splittability of All Extensions}

Every subalgebra of the algebra $A S L(2, \mathbf{R})$ is conjugate to one of the following algebras:

$$
0, \quad\langle D>, \quad\langle T>, \quad\langle S+T>, \quad\langle D, T>, \quad A S L(2, \mathbf{R}) .
$$

When we speak about subalgebras of the algebra $\operatorname{ASL}(2, \mathbf{R})$, we mean the subalgebras (3). Denote by $\pi$ the projection of $A \bar{G}_{4}(n)$ onto $B=A O(n) \oplus A G L(2, \mathbf{R})$. Let $F$ be a subalgebra of $B$, and let $F^{\prime}$ be a subalgebra of the algebra $A \bar{G}_{4}(n)$ such that $\pi\left(F^{\prime}\right)=F$. If every sybalgebra $F^{\prime}$ is splittable, we shall say that $F$ has only splittable extensions in the algebra $A \bar{G}_{4}(n)$.

Theorem 1 Let $\sigma, \tau$ and $\omega$ be projections of $B$ onto $A G L(2, \mathbf{R}), A S L(2, \mathbf{R})$ and $A O(n)$, respectively. The subalgebra $F$ of the algebra $B$ has only splittable extensions in $A \bar{G}_{4}(n)$ if and only if one of the following conditions is satisfied: (1) $\sigma(F)$ coincides with one of the algebras $\langle D+3 Z\rangle,\langle S+T+\alpha Z\rangle,\langle T+\alpha Z\rangle$, where $\alpha \neq 0$; (2) $\sigma(F)$ contains 
$Z$ or $D+\alpha Z$, where $\alpha \notin\{-1,1,3\}$; (3) $\sigma(F)=<S+T>$ and $F$ is not conjugate under $\operatorname{Ad}(A O(n) \oplus A S L(2, \mathbf{R}))$ to any subdirect sum of $\left\langle J_{12}+S+T\right\rangle$ and a subalgebra of the algebra $A O[3, n] ;(4) \tau(F) \neq<S+T>$ and $\omega(F)$ is not conjugate under $A d A O(n)$ to any subalgebra of the algebra $A O(n-1) ;(5) \sigma(F)=0$ and $\omega(F)$ is a semisimple algebra.

\section{The Structure of Splitting Subalgebras}

In this section, we use the concepts of irreducible and primary parts of an orthogonal algebra. These definitions can be found in [5,7].

Let $L_{1}, L_{2}, \ldots L_{q}$ be primary parts of a nonzero subalgebra $K^{\prime}$ of the algebra $A O(n)$, let $K^{\prime \prime}$ be a subalgebra of the algebra $A G L(2, \mathbf{R})$, whose projection onto $A S L(2, \mathbf{R})$ is different from $\langle S+T\rangle$, and let $K$ be a subdirect sum of $K^{\prime}$ and $K^{\prime \prime}$. Moreover, let $d_{0}=0<d_{1}<\ldots<d_{q}$ be a sequence of integers. $L_{i}$ is a subalgebra of $A O\left[d_{i-1}+1, d_{i}\right]$, not conjugate to any subalgebra of the algebra $A O\left[d_{i-1}+1, d_{i}-1\right] . U$ is a subspace of the space $Q[1, n]$, and $U_{i}$ is the projection of $U$ onto $Q\left[d_{i-1}+1, d_{i}\right] \quad(i=1, \ldots, q) ; U_{q+1}$ is the projection of $U$ onto $Q\left[d_{q}+1, n\right]$ if $d_{q}<n$ and $U_{q+1}=0$ for $d_{q}=n$.

Theorem 2 If $U$ is invariant under $K$, then $U=U_{1} \oplus \cdots \oplus U_{q} \oplus U_{q+1}$ and each subspace $U_{i}(i=1, \ldots, q+1)$ is invariant under each algebra $L_{j} \oplus K^{\prime \prime}(j=1, \ldots, q)$.

Because of Theorem 2, the study of splittable subalgebras of the algebra $A \bar{G}_{4}(n)$, whose projections onto $A S L(2, \mathbf{R})$ differ from $\langle S+T\rangle$, is reduced to the study of splittable subalgebras of the form $U \rightrightarrows\left(L^{\prime} \oplus L^{\prime \prime}\right)$, where $L^{\prime}$ is a primary subalgebra of the algebra $A O(n), L^{\prime \prime}$ a subalgebra of the algebra $A S L(2, \mathbf{R})$ and $U$ a subspace of $Q[1, n]$.

Let $\mu_{a}$ be the projection of $Q[1, n]$ onto $\left\langle G_{a}, P_{a}\right\rangle$. If $U$ is a nonzero subspace of the space $Q[1, n]$, then the index of $U$, ind $U$, is the set of all $a \in\{1, \ldots, n\}$ such that $\mu_{a}(U) \neq 0$. The decomposition of the space $U$ into the sum of the spaces $U_{1}, \ldots, U_{m}$ is said to be index-direct if ind $U_{a} \cap i n d U_{b}=\emptyset$ whenever $a \neq b$ for all $a, b=1, \ldots, m$.

Theorem 3 Let $K$ be a primary subalgebra of the algebra $A O(n)$ which is a subdirect sum of irreducible subalgebras of the algebras $A O[1, a], \quad A O[a+1,2 a], \ldots$, $A O[(r-1) a+1, r a]$, respectively. Let $L$ be a subalgebra of the algebra $A S L(2, \mathbf{R})$ and let $U$ be a nonzero subspace of the space $Q[1, r a]$ which is invariant under $K \oplus L$. If $L=<D>$, then there exists an automorphism $\psi \in A d A O(n)$ such that $\psi(K \oplus L)=K \oplus L$ and $\psi(U)$ is an index-direct sum of subspaces of the form

$$
\begin{aligned}
& V[k a+1,(k+1) a], W[k a+1,(k+1) a], Q[k a+1,(k+1) a], \\
& V[k a+1,(k+1) a] \oplus<P_{k a+1}+\lambda P_{(k+1) a+1}, \ldots, P_{(k+1) a}+\lambda P_{(k+2) a}>.
\end{aligned}
$$

If $L=<T>$, then (up to an automorphism from $A d A O(n)$ ) the space $U$ is an index-direct sum of the spaces of the form

$$
\begin{aligned}
& W[k a+1,(k+1) a], Q[k a+1,(k+1) a], \\
& W[k a+1,(k+1) a] \oplus<G_{k a+1}+\lambda P_{(k+1) a+1}, \ldots, G_{(k+1) a}+\lambda P_{(k+2) a}>.
\end{aligned}
$$


If $L=<D, T>$, then (up to an automorphism from $A d A O(n)$ ) the space $U$ coincides with one of the following subspaces

$$
W[1, d a], \quad Q[1, d a], \quad Q[1, b a] \oplus W[b a+1, d a] \quad(1 \leq d \leq r) .
$$

If $L=<D, S, T>$, then (up to an automorphism from $A d A O(n)$ ) the space $U$ coincides with $Q[1, d a]$, where $1 \leq d \leq r$.

Theorems 2 and 3 follow immediately from Theorems III.4.1-III.4.3 [7].

Theorem 4 Let $K$ be a primary subalgebra of the algebra $A O(n)$ which is a subdirect sum of the algebras $A O[1, a], A O[a+1,2 a], \ldots, A O[(r-1) a+1, r a]\left(1 \leq r \leq\left[\frac{n}{a}\right]\right)$. The nonzero subspaces of the space $Q[1, r a]$ invariant under $K \oplus<S+T>$ are (up to $\operatorname{Ad} A O(n)$ conjugation) the following subspaces:

$$
\begin{aligned}
& Q[1, d a](d=1, \ldots, r), \quad U_{m}(m=1, \ldots, r), \\
& U_{m}+Q[m a+1, d a](m=1, \ldots, r-1 ; d=m+1, \ldots, r),
\end{aligned}
$$

where $U_{m}$ is a subdirect sum of the spaces $V[1, m a]$ and $W[1, m a]$, which has zero intersections with these spaces. If $a>2$, then $m$ is an even number and there exists an automorphism $\psi \in A d A \bar{G}_{3}(n)$ such that $\psi(K \oplus<S+T>)=K \oplus<S+T>$ and $\psi\left(U_{m}\right)$ is an index-direct sum of subspaces of the form

$$
\begin{aligned}
& <G_{l a+1}-\lambda^{-1} P_{(l+1) a+1}, \ldots, G_{(l+1) a}-\lambda^{-1} P_{(l+2) a}, \\
& G_{(l+1) a+1}+\lambda P_{l a+1}, \ldots, G_{(l+2) a}+\lambda P_{(l+1) a},(0<|\lambda| \leq 1) .
\end{aligned}
$$

If $a=2$, then there exists an automorphism $\psi \in A d A \bar{G}_{3}(n)$ such that $\psi(K \oplus<S+$ $+T\rangle)=K \oplus\langle S+T\rangle$ and $\psi\left(U_{m}\right)$ is an index-direct sum of subspaces of the form

$$
<G_{2 l+1}-\gamma P_{2 l+2}, G_{2 l+2}+\gamma P_{2 l+1}>(\gamma= \pm 1)
$$

and

$$
G_{2 l+1}-\lambda^{-1} P_{2 l+3}, G_{2 l+2}-\lambda^{-1} P_{2 l+4}, G_{2 l+3}+\lambda P_{2 l+1}, G_{2 l+4}+\lambda P_{2 l+2}>,
$$

where $0<|\lambda| \leq 1$.

\section{Conjugacy of Subalgebras When the Group of Automorphisms is Extended}

We denote by $\tau$ the projection of the algebra $A \bar{G}_{4}(n)$ onto $A S L(2, \mathbf{R})$.

Theorem 5 Let $L_{1}, L_{2}$ be subalgebras of the algebra $A \bar{G}_{4}(n)$ such that $\tau\left(L_{1}\right)=\tau\left(L_{2}\right)$. If the subalgebras $L_{1}$ and $L_{2}$ are conjugate under $A d A \bar{G}_{4}(n)$, then they are conjugate under $A d F$, where $F=A G_{0}(n) \boxplus K$ with

(1) $K=<D, T, Z>$ for $\tau\left(L_{1}\right)=<T>$ or $\tau\left(L_{1}\right)=<D, T>$

(2) $K=<S+T, Z>$ for $\tau\left(L_{1}\right)=<S+T>$. 
Let $A P(1, n)=<P_{0}, P_{1}, \ldots, P_{n}>\oplus<J_{\alpha \beta}: \alpha, \beta=0,1, \ldots, n>$ be the Poincaré algebra realized as the algebra of real matrices of order $n+2$. It contains the algebra $L=A G_{1}(n-1) \boxplus\left\langle J_{0 n}\right\rangle$, where $A G_{1}(n-1)$ is the classical Galilei algebra, with $M=$ $P_{0}+P_{n}, T=\frac{1}{2}\left(P_{0}-P_{n}\right), G_{a}=J_{0 a}-J_{a n}(a=1, \ldots, n-1)$.

Theorem 6 Let $L_{1}, L_{2}$ be subalgebras of the algebra $A G_{1}(n-1)$. These subalgebras are conjugate under $\operatorname{Ad} A P(1, n)$ if and only if they are $A d L$-conjugate or when there exist automophisms $\psi_{1}, \psi_{2} \in A d L$ with $\psi_{1}\left(L_{1}\right)=C\left[\psi_{2}\left(L_{2}\right)\right] C^{-1}$ where $C=\operatorname{diag}[1,-1,1, \ldots$ $\ldots, 1,1,-1]$.

Corollary Let $L_{1}, L_{2}$ be subalgebras of the algebra $A G_{1}(n-1)$ which are not conjugate under $A d A G_{1}(n-1)$ to subalgebras of $<M, T, P_{1}, \ldots, P_{n-1}>\nexists A O(n-1)$. Then the algebras $L_{1}, L_{2}$ are $A d A P(1, n)$-conjugate if and only if they are $A d L$-conjugate.

Suppose that the conformal algebra $A C(1, n), n \geq 2$, is realized as the algebra $A O(2, n+$ $1)$ of real matrices of order $n+3$. Let $P_{\alpha}, K_{\alpha}, D, J_{\alpha \beta}(\alpha, \beta=0,1, \ldots, n)$ be the standard basis of $A C(1, n)$. The algebra $A C(1, n)$ contains the full Galilei algebra $A G_{4}(n-1)$, with $M=P_{0}+P_{n}, G_{a}=J_{0 a}-J_{a n}(a=1, \ldots, n-1), D^{\prime}=-\left(J_{0 n}+D\right), S=\frac{1}{2}\left(K_{0}+K_{n}\right)$, $T=\frac{1}{2}\left(P_{0}-P_{n}\right), Z=J_{0 n}-D$.

Theorem 7 Let $L_{1}, L_{2}$ be subalgebras of $A G_{4}(n-1)$. These subalgebras are conjugate under $A d A C(1, n)$ if and only if they are conjugate under $A d A G_{4}(n-1)$ or when there exist automorphisms $\psi_{1}, \psi_{2} \in A d A G_{4}(n-1)$ with $\psi_{1}\left(L_{1}\right)=C\left[\psi_{2}\left(L_{2}\right)\right] C^{-1}$, where $C$ is a matrix of order $n+3$, equal to diag $[1, \ldots, 1,-1,-1]$ or diag $[1,1,-1,1, \ldots, 1,-1,1]$.

\section{References}

[1] Sorba P., The Galilei group and its connected subgroups, J. Math. Phys., 1976, V.17, N 6, 941-953.

[2] Burdet G., Patera J., Perrin M., Winternitz P., Sous-algèbres de Lie de l'algèbre de Schrödinger, Ann. Sc. Math. Quebec., 1978, V.2, N 1, 81-108.

[3] Burdet G., Patera J., Perrin M., Winternitz P., The optical group and its subgroups, J.Math.Phys., 1978, V.19, N 8, 1758-1780.

[4] Fushchych W.I., Barannyk A.F. and Barannyk L.F., Continuous subgroups of the generalized Galilei group. I, Kiev, Institute of Mathematics, Academy of Sciences of Ukrainian SSR; 85.19, Preprint, 1985, 46p.

[5] Barannyk L.F., Fushchych W.I., On continuous subgroups of the generalized Schrödinger groups, J. Math. Phys., 1989, V.30, N 2, 280-290.

[6] Gagnon L., Continuous subgroups of the Galilei and Galilei-similitude groups, Can. J. Phys., 1989, V.67, 1-24.

[7] Fushchych W.I., Barannyk L.F. and Barannyk A.F., Subgroup Analysis of the Galilei and Poincaré Groups and Reduction of Nonlinear Equations, Kiev, Naukova Dumka, 1991.

[8] Barannyk L.F., Barannyk A.F., Subalgebras of the generalized Galilei algebra, Group-Theoretical Studies of Equations of Mathematical Physics, Kiev, Mathematical Institute, 1985, 39-43.

[9] Barannyk L.F., Subalgebras of the generalized extended Galilei algebra, Ukr. Math. J., 1988, V.40, N 6, 705-709.

[10] Barannyk L.F., Marchenko V.A., On invariant solutions of the multidimensional heat equation, Dopovidi Akademii Nauk Ukrainy (Proceedings of the Nat. Academy of Sciences of Ukraine), 1995, V.3, $12-15$.

[11] Barannyk A.F., Marchenko V.A. and Fushchych W.I., On the reduction and exact solutions of the nonlinear multidimensional Schrödinger equations, Theor. Math. Phys., 1991, V.87, N 2, 220-234.

[12] Basarab-Horwath P., Barannyk L.F. and Fushchych W.I. New solutions of the wave equation by reduction to the heat equation, J. Phys. A: Math. Gen., 1995, V.28, 1-14. 